withdrawal studies may employ more personalized, science-based decision making, stratifying patients according to validated assessments for their propensity for tolerance. As well, these findings provide a basis for new studies in both humans and animal models to better understand solid organ transplant tolerance and the important and unique contributions that the liver, an immune organ itself, makes to the tolerance process following liver transplantation.

Address correspondence to: Vicki Seyfert-Margolis, Immune Tolerance Network, 3 Bethesda Metro Center, Suite 400, Bethesda, Maryland 20814, USA. Phone: (240) 235-6133; Fax: (240) 235-6198; E-mail: vseyfert@immunetolerance.org.

1. Martínez-Llordella, M., et al. 2008. Using transcriptional profiling to develop a diagnostic test of operational tolerance in liver transplant recipients. J. Clin. Invest. 118:2845-2857.

2. Racanelli, V., and Rehermann, B. 2006. The liver as an immunological organ. Hepatology. 43:S54-S62.

3. Crispe, I.N. 2003. Hepatic T cells and liver toler- ance. Nat. Rev. Immunol. 3:51-62.

4. Starzl, T.E., et al. 1993. Cell migration and chimerism after whole-organ transplantation: the basis of graft acceptance. Hepatology. 17:1127-1152.

5. Mazariegos, G.V., et al. 1997. Weaning of immunosuppression in liver transplant recipients. Transplantation. 63:243-249.

6. Devlin, J., et al. 1998. Defining the outcome of immunosuppression withdrawal after liver transplantation. Hepatology. 27:926-933.

7. Koshiba, T., et al. 2007. Clinical, immunological, and pathological aspects of operational tolerance after pediatric living-donor liver transplantation. Transpl. Immunol. 17:94-97.

8. Takatsuki, M., et al. 2001. Analysis of alloreactivity and intragraft cytokine profiles in living donor liver transplant recipients with graft acceptance. Transpl. Immunol. 8:279-286.

9. Li, Y., et al. 2004. Analyses of peripheral blood mononuclear cells in operational tolerance after pediatric living donor liver transplantation. Am. J. Transplant. 4:2118-2125.

10. Martínez-Llordella, M., et al. 2007. Multiparameter immune profiling of operational tolerance in liver transplantation. Am. J. Transplant. 7:309-319.

11. Abo, T., Kawamura, T., and Watanabe, H. 2000. Physiological responses of extrathymic T cells in the liver. Immunol. Rev. 174:135-149.

12. McIntyre, K.W., and Welsh, R.M. 1986. Accumulation of natural killer and cytotoxic T large granular lymphocytes in the liver during virus infection. J. Exp. Med. 164:1667-1681.
13. Toyabe, S., et al. 1997. Requirement of IL-4 and liver NK1+ T cells for concanavalin A-induced hepatic injury in mice. J. Immunol. 159:1537-1542.

14. Vicari, A.P., Mocci, S., Openshaw, P., O'Garra, A., and Zlotnik, A. 1996. Mouse gamma delta $\mathrm{TCR}+\mathrm{NK} 1.1+$ thymocytes specifically produce interleukin-4, are major histocompatibility complex class I independent, and are developmentally related to alpha beta TCR+NK1.1+ thymocytes. Eur. J. Immunol. 26:1424-1429.

15. Knolle, P.A., et al. 1999. Induction of cytokine production in naive CD4(+) T cells by antigen-presenting murine liver sinusoidal endothelial cells but failure to induce differentiation toward Th1 cells. Gastroenterology. 116:1428-1440.

16. Limmer, A., et al. 2000. Efficient presentation of exogenous antigen by liver endothelial cells to CD8+ T cells results in antigen-specific T-cell tolerance. Nat. Med. 6:1348-1354.

17. Levine, A.J. 1997. p53, the cellular gatekeeper for growth and division. Cell. 88:323-331.

18. Levkau, B., et al. 1998. Cleavage of p21Cip1/Waf1 and p27Kip1 mediates apoptosis in endothelial cells through activation of $\mathrm{Cdk} 2$ : role of a caspase cascade. Mol. Cell. 1:553-563.

19. Kawai, T., et al. 2008. HLA-mismatched renal transplantation without maintenance immunosuppression. N. Engl. J. Med. 358:353-361.

20. Brouard, S., et al. 2007. Identification of a peripheral blood transcriptional biomarker panel associated with operational renal allograft tolerance. Proc. Natl. Acad. Sci. U. S. A. 104:15448-15453.

\title{
Immune response hinders therapy for lysosomal storage diseases
}

\author{
Katherine P. Ponder \\ Department of Internal Medicine and Department of Biochemistry and Molecular Biophysics, \\ Washington University School of Medicine, St. Louis, Missouri, USA.
}

\begin{abstract}
Enzyme replacement therapy (ERT) for the lysosomal storage disease mucopolysaccharidosis I (MPS I) involves i.v. injection of $\alpha$-L-iduronidase, which can be taken up by cells throughout the body. While a significant immune response to ERT has been shown in patients with MPS I, little is known about what effect anti-enzyme antibodies have on treatment efficacy. In this issue of the JCI, Dickson et al. demonstrate that anti-enzyme antibodies inhibit enzyme uptake and substantially limit the therapeutic efficacy of ERT in canines with MPS I (see the related article beginning on page 2868). Furthermore, the induction of immune tolerance - via oral delivery of cyclosporine $A$ and azathioprine for two months at the time of initiation of ERT with recombinant human $\alpha$-L-iduronidase - improved enzyme uptake in organs. Therefore, transient immunosuppression may enhance ERT for lysosomal storage diseases.
\end{abstract}

Nonstandard abbreviations used: ERT, enzyme replacement therapy; GAG, glycosaminoglycan; IDUA, $\alpha$-L-iduronidase; LSD, lysosomal storage disease; M6P, mannose 6-phosphate; MPS I, mucopolysaccharidosis I.

Conflict of interest: The author has declared that no conflict of interest exists.

Citation for this article: J. Clin. Invest. 118:2686-2689 (2008). doi:10.1172/JCI36521.
Intravenous injection of recombinant proteins can treat genetic deficiencies such as lysosomal storage diseases (LSD) or hemophilia. Since the patient does not produce the normal protein, the therapeutic protein can be recognized as foreign and induce antibodies. It has been known for decades that antibodies that inhibit the coagula- tion function of Factor VIII develop in $30 \%$ of patients with severe hemophilia A (1). These antibodies are known as inhibitors and bind to epitopes of Factor VIII that are very important for its coagulation function. However, it has been less clear how antibodies may affect the efficacy of recombinant proteins that are injected i.v. to treat LSDs. The study by Dickson et al. in this issue of the JCI (2) demonstrates that antibodies reduce the efficacy of this therapeutic approach for the LSD mucopolysaccharidosis I (MPS I) and identifies an immunosuppressive regimen that is highly effective at blocking antibody formation when given at the time of initiation of protein therapy in dogs with MPS I.

\section{Enzyme replacement therapy for LSDs}

LSDs are due to a deficiency in any of several enzymes that degrade various sub- 


\section{Table 1}

Summary of the effect of antibodies during ERT for LSD

\begin{tabular}{|c|c|c|c|}
\hline Disease & Deficient protein & Target cells & Effect of antibodies on ERT \\
\hline Gaucher & Acid $\beta$-glucosidase & Macrophages & Usually has little effect in patients (4) \\
\hline MPS I & IDUA & $\begin{array}{l}\text { Hepatocytes, renal tubular cells, splenocytes, } \\
\text { neurons, fibroblasts, other }\end{array}$ & $\begin{array}{c}\text { Inhibits delivery to tissues } \\
\text { in MPS I dogs (2) }\end{array}$ \\
\hline MPS II & Iduronate sulfatase & Similar to MPS I & Not reported to reduce efficacy \\
\hline MPS VI & $\begin{array}{l}N \text {-acetyl-galactosamine } \\
\text { 4-sulfatase }\end{array}$ & $\begin{array}{c}\text { Similar to MPS I, except neurons have less storage } \\
\text { and chondrocytes have more storage }\end{array}$ & Reduces efficacy in cats (17) \\
\hline Pompe & Acid $\alpha$-glucosidase & Skeletal and cardiac myocytes & $\begin{array}{l}\text { Reduces efficacy in mice (18) and } \\
\text { may reduce efficacy in humans (19) }\end{array}$ \\
\hline Fabry & $\alpha$-Galactosidase & Vascular cells, kidney & Reduces efficacy in mice (20) \\
\hline
\end{tabular}

strates. Although most of the enzyme in a normal cell derives from de novo synthesis and translocation to the lysosome, enzyme can also be taken up from outside the cell. This process has served as the basis for a treatment called enzyme replacement therapy (ERT), in which enzyme is injected i.v., diffuses to cells in many parts of the body, and is taken up via a receptor and translocated to the lysosome. The first LSD that was treated with ERT was Gaucher disease, which is due to deficiency of the lysosomal acid $\beta$-glucosidase. It was demonstrated in the early 1990s that purified enzyme that was modified with mannose residues could be taken up by macrophages via the mannose receptor and reduce the clinical manifestations in patients with the nonneuropathic form of Gaucher disease (3). Although antibodies developed in approximately $15 \%$ of patients, they did not usually affect the efficacy of treatment (4). This may reflect the fact that macrophages are the target cell, and macrophages contain IgG receptors that could take up the enzyme present in immune complexes.

ERT has been developed more recently for several other LSDs, including MPS I, MPS II, MPS VI, Fabry disease, and Pompe disease (5). For all of these diseases, enzyme must be taken up by cells other than macrophages for an appropriate therapeutic effect to be achieved, as summarized in Table 1. For this reason, the enzymes used for all of these disorders have been modified with mannose 6-phosphate (M6P), as the M6P receptor is present on most cells. Antibodies have developed in several studies, but their effect on ERT has been somewhat unclear.

\section{Anti-IDUA antibodies develop after ERT for MPS I}

MPS I is due to deficiency in $\alpha$-L-iduronidase (IDUA) and results in the accumula- tion of the glycosaminoglycans (GAGs) dermatan sulfate and heparan sulfate. In the initial study of ERT with IDUA for MPS I, only $40 \%$ of patients developed anti-IDUA antibodies, and these did not appear to alter the clinical effect (6). However, none of these initial patients had IDUA-null mutations. In a more recent study in which $70 \%$ of the patients had IDUA-null mutations, all patients developed anti-IDUA antibodies, and those with anti-IDUA antibody titers that were greater than 1:10,000 had relatively modest reductions in urine GAG levels when compared with the reduction in patients with low or undetectable titers of anti-IDUA antibodies (7). Although these results suggest that anti-IDUA antibodies reduce the efficacy of ERT for MPS I, the critical test is whether enzyme is delivered to tissues and can reduce the biochemical, pathological, and clinical manifestations of disease. It is difficult to assess this response in patients, as biopsies of organs or tissues cannot readily be performed.

\section{Anti-IDUA antibodies reduce the efficacy of ERT for MPS I}

The current study by Dickson et al. (2) evaluates the effect of anti-IDUA antibody formation on disease manifestations in MPS I dogs that received ERT with recombinant human IDUA. This group had previously demonstrated that MPS I dogs that received i.v. injection of M6P-modified recombinant human IDUA consistently developed antiIDUA antibodies $(8,9)$, but the significance of this antibody production was unclear. In their current article (2), the authors compare the effect of ERT on disease manifestations in MPS I dogs with high titers of antiIDUA antibodies with that seen in MPS I dogs that were tolerized to recombinant human IDUA using the regimen detailed below. They demonstrate that high titers of
anti-IDUA antibodies reduced the uptake of IDUA by MPS I fibroblasts to less than $10 \%$ of that seen with serum from animals with low or negative titers of antibodies. Furthermore, anti-IDUA antibodies reduced delivery of enzyme to organs with low levels of macrophages and reduced the ability of ERT to lower GAG levels. For example, IDUA enzyme activity was 2.4- and 5.1-fold higher, respectively, in heart valves and the renal medulla in tolerant dogs than in dogs with high-titer antibodies after administration of the standard dose of $0.58 \mathrm{mg} / \mathrm{kg} / \mathrm{wk}$ of recombinant human IDUA, while the pathological abnormalities in heart valves and renal medulla, respectively, were only $24 \%$ and $60 \%$ as severe in tolerized dogs compared with dogs with high-titer antibodies. Furthermore, although a 3.4-foldhigher dose of $2 \mathrm{mg} / \mathrm{kg} / \mathrm{wk}$ further reduced pathological evidence of lysosomal storage in tolerized MPS I dogs, this higher dose was not as effective in animals with hightiter antibodies. These data provide compelling evidence that anti-IDUA antibodies adversely affect the efficacy of ERT. The anti-IDUA antibody probably binds to an epitope at or near the M6P modification and sterically inhibits the protein from binding to the M6P receptor and being taken up by the cell, as diagrammed in Figure 1 . This study also suggests that the dose of enzyme that is currently used for ERT is too low to be effective at some sites. Early studies to identify the appropriate dose relied upon reduction in lysosomal storage in easy-to-treat organs such as liver and spleen (6), and these doses may need to be revised based on these data.

\section{Immunosuppressive regimen used to induce tolerance}

The immunosuppressive regimen used to tolerize MPS I dogs was remarkable for its 
A Patients without anti-IDUA antibodies

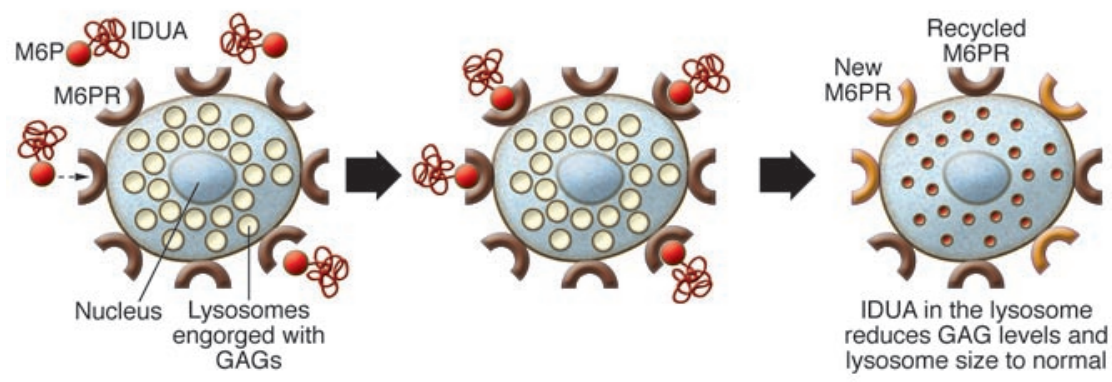

B Patients with anti-IDUA antibodies

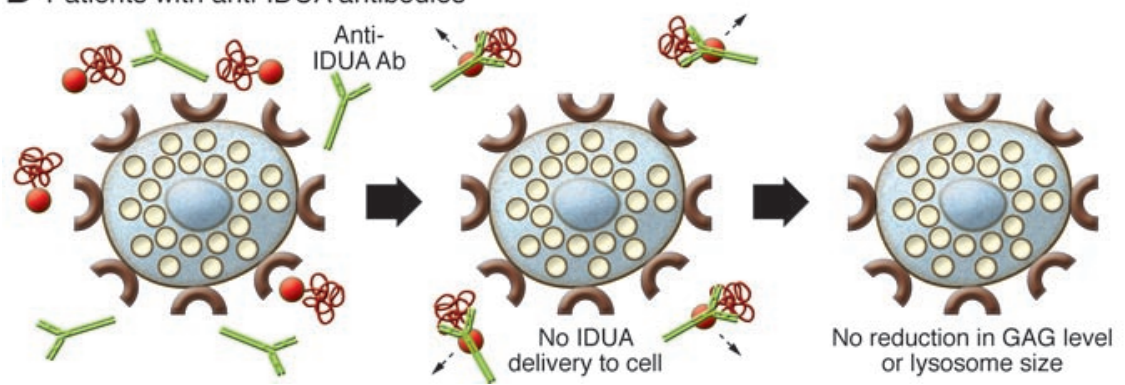

Figure 1

Anti-IDUA antibodies can inhibit the uptake of the therapeutic enzyme IDUA during enzyme replacement therapy for the lysosomal storage disease MPS I. (A) Effect of ERT in MPS I patients without anti-IDUA antibodies. In the LSD MPS I, deficiency in the enzyme IDUA results in the accumulation of GAGs within lysosomes in patient cells. ERT involves i.v. injection of M6P-modified IDUA that can diffuse to cells and bind to the M6P receptor (M6PR) on the cell surface. M6PR can be internalized with the IDUA and translocate the enzyme to the lysosome, resulting in GAG degradation and a reduction in lysosome size. M6PR can then be recycled to the cell surface or replaced with new receptor. (B) Effect of ERT in patients with anti-IDUA antibodies. In some MPS I patients, a significant immune response to ERT has been shown; however, little was known about the effect of anti-enzyme antibodies on treatment outcome. In this issue of the JCl, Dickson et al. (2) show that in dogs with MPS I receiving IDUA via ERT, anti-IDUA antibodies may develop and bind to IDUA and sterically block MP6 from binding M6PR. This results in a failure of the enzyme to reach the lysosome and lack of a therapeutic effect. The authors go on to demonstrate that the induction of IDUA-specific immune tolerance in these animals, compared with dogs that were not tolerized to IDUA, improved IDUA uptake, increased GAG degradation, and reduced tissue pathology. Therefore, transient immunosuppression may enhance ERT for LSDs.

high success rate, as $100 \%$ of 14 MPS I dogs that were treated in this (2) and a previous (10) study were successfully tolerized. Although the initial dose of cyclosporine A $(25 \mathrm{mg} / \mathrm{kg})$ was approximately 3 -fold greater than the average dose used for organ transplantation and the initial target cyclosporine trough level in blood was relatively high, at $400 \mathrm{ng} / \mathrm{ml}$, the dose was reduced to $12.5 \mathrm{mg} / \mathrm{kg}$ after 1 month, 6.25 $\mathrm{mg} / \mathrm{mg}$ after 1.5 months, and was discontinued after 2 months. The starting dose of azathioprine $(5 \mathrm{mg} / \mathrm{kg})$ every other day was approximately $50 \%$ of the dose used for renal transplantation and was similarly reduced to $50 \%$ and $25 \%$ of the initial dose after 1 and 1.5 months, respectively, and then discontinued after 2 months. Thus, the immunosuppressive regimen was relatively mild, and indeed, no serious adverse effects were reported in any animals.

Although this approach appeared to be extraordinarily effective in MPS I dogs, the choice of cyclosporine A was a bit surpris- ing, as some studies have demonstrated that cyclosporine A blocks activation-induced cell death of effector T cells and is detrimental to tolerance induction by costimulation blockade (11-14). Azathioprine appears to be a more logical choice, as it has been reported to block CD28-dependent costimulatory signaling (15). Indeed, although administration of cyclosporine A with azathioprine induced tolerance in 1 of 1 dogs that received the enzyme deficient in Pompe disease (acid $\alpha$-glucosidase), an identical regimen was not effective in mice with Pompe disease (16). In addition, this regimen was not effective at inducing tolerance to soluble proteins that did not contain M6P, such as ovalbumin or IDUA that had been enzymatically treated to remove the M6P. Further investigation will clearly be required to identify the mechanism by which this particular regimen induced tolerance to M6P-modified IDUA, to test its efficacy for inducing tolerance to other proteins, and to determine whether it can prevent antibody formation in humans.
In conclusion, the current study by Dickson et al. (2) demonstrates that antibodies against recombinant human IDUA can reduce the efficacy of ERT for MPS I in dogs. Importantly, the study identifies a highly effective and transient immunosuppressive regimen that can prevent antibody formation in $100 \%$ of dogs without any overt adverse effects. It is likely that this or other immunosuppressive protocols will become routine at the initiation of ERT for LSD or of protein therapy for other genetic diseases, as patients with null mutations will likely be at high risk for antibody formation. Thus, for these disorders, a stitch in time saves nine in our efforts to provide an effective treatment for these patients.

Address correspondence to: Katherine P. Ponder, Washington University School of Medicine, 660 S. Euclid Avenue, St. Louis, Missouri 63110, USA. Phone: (314) 362-5188; Fax: (314) 362-8813; E-mail: KPONDER@DOM.WUSTL.EDU. 
1. Fakharzadeh, S.S., and Kazazian, H.H., Jr. 2000. Correlation between factor VIII genotype and inhibitor development in hemophilia A. Semin. Thromb. Hemost. 26:167-171.

2. Dickson, P., et al. 2008. Immune tolerance improves the efficacy of enzyme replacement therapy in canine mucopolysaccharidosis I. J. Clin. Invest. 118:2868-2876.

3. Barton, N.W., et al. 1991. Replacement therapy for inherited enzyme deficiency - macrophagetargeted glucocerebrosidase for Gaucher's disease. N. Engl. J. Med. 324:1464-1470.

4. Starzyk, K., Richards, S., Yee, J., Smith, S.E., and Kingma, W. 2007. The long-term international safety experience of imiglucerase therapy for Gaucher disease. Mol. Genet. Metab. 90:157-163.

5. Rohrbach, M., and Clarke, J.T. 2007. Treatment of lysosomal storage disorders: progress with enzyme replacement therapy. Drugs. 67:2697-2716.

6. Kakkis, E.D., et al. 2001. Enzyme-replacement therapy in mucopolysaccharidosis I. N. Engl.J. Med. 344:182-188

7. Wraith, J.E., et al. 2007 Enzyme replacement therapy in patients who have mucopolysaccharidosis I and are younger than 5 years: results of a multinational study of recombinant human alpha-L-iduronidase (laronidase). Pediatrics. 120:e37-e46.

8. Shull, R.M., et al. 1994. Enzyme replacement in a canine model of Hurler syndrome. Proc. Natl. Acad. Sci. U. S. A. 91:12937-12941.

9. Kakkis, E.D., et al. 1996. Long-term and high-dose trials of enzyme replacement therapy in the canine model of mucopolysaccharidosis I. Biochem. Molec. Med. 58:156-167.

10. Kakkis, E., et al. 2004. Successful induction of immune tolerance to enzyme replacement therapy in canine mucopolysaccharidosis I. Proc. Natl. Acad. Sci. U. S. A. 101:829-834.

11. Gao, W., et al. 2007. Contrasting effects of cyclosporine and rapamycin in de novo generation of alloantigen-specific regulatory T cells. Am. J. Transplant. 7:1722-1732.

12. Lim, D.G., et al. 2007. Effect of immunosuppressants on the expansion and function of naturally occurring regulatory $\mathrm{T}$ cells. Transpl. Immunol. 18:94-100.

13. Adams, A.B., Pearson, T.C., and Larsen, C.P. 2001. Conventional immunosuppression and co-stimulation blockade. Philos. Trans. R. Soc. Lond. B Biol. Sci. 356:703-705.

14. San Segundo, D., Fábrega, E., López-Hoyos, M., and Pons, F. 2007. Reduced numbers of blood natural regulatory $T$ cells in stable liver transplant recipients with high levels of calcineurin inhibitors. Transplant Proc. 39:2290-2292.

15. Tiede, I., et al. CD28-dependent Rac1 activation is the molecular target of azathioprine in primary human CD4+ T lymphocytes. J. Clin. Invest. 111:1133-1145

16. Joseph, A., Munroe, K., Housman, M., Garman, R., and Richards, S. 2008. Immune tolerance induction to enzyme-replacement therapy by co-administration of short-term, low-dose methotrexate in a murine Pompe disease model. Clin. Exp. Immunol. 152:138-146.

17. Turner, C.T., Hopwood, J.J., Bond, C.S., and Brooks, D.A. 1999. Immune response to enzyme replacement therapy: 4-sulfatase epitope reactivity of plasma antibodies from MPS VI cats. Mol. Genet. Metab. 67:194-205.

18. Sun, B., Bird, A., Young, S.P., Kishnani, P.S., Chen, Y.T., and Koeberl, D.D. 2007. Enhanced response to enzyme replacement therapy in Pompe disease after the induction of immune tolerance. Am. J. Hum. Genet. 81:1042-1049.

19. Kishnani, P.S., et al. 2007. Recombinant human acid [alpha]-glucosidase: major clinical benefits in infantile-onset Pompe disease. Neurology. 68:99-109.

20. Ohashi, T., Iizuka, S., Ida, H., and Eto, Y. 2008. Reduced alpha-Gal A enzyme activity in Fabry fibroblast cells and Fabry mice tissues induced by serum from antibody positive patients with Fabry disease. Mol. Genet. Metab. 94:313-318.

\title{
It takes two to tango: cigarette smoke partners with viruses to promote emphysema
}

\author{
Rubin M. Tuder and Jeong H. Yun
}

Program in Translational Lung Research, Division of Pulmonary Sciences and Critical Care Medicine, Department of Medicine, University of Colorado Denver School of Medicine, Denver, Colorado, USA.

\begin{abstract}
Viruses constitute a constant and renewed threat to humans. Not only do viruses cause disease directly due to their tissue tropism and pathogenicity, but they have also been linked to autoimmunity. In their study in this issue of the JCI, Kang et al. show that exposure to cigarette smoke induces alterations in the innate immune response to viral infection and that these changes hasten alveolar destruction characteristic of emphysema in mice (see the related article beginning on page 2771). This study builds on evidence that patients with chronic obstructive pulmonary disease have clinical exacerbations associated with viral or bacterial infections, which lead to worsened lung function and increased mortality. This novel paradigm may aid related genetic, biomarker, and therapeutic developments and provides important insights into the pathogenesis of emphysematous lung destruction.
\end{abstract}

The worldwide magnitude of diseases caused by cigarette smoking has outweighed

Nonstandard abbreviations used: COPD, chronic obstructive pulmonary disease; dsRNA, double-stranded RNA; eIF2 $\alpha$, eukaryotic initiation factor- $\alpha$; MAVS, mitochondrial antiviral signaling; PAMP, pathogenassociated molecular pattern; PKR, RNA-dependent protein kinase; poly(I:C), polyinosinedeoxycytidylic acid; RIG-1, retinoic acid-inducible gene-I; RLH, RIG-1-like helicase.

Conflict of interest: The authors have declared that no conflict of interest exists.

Citation for this article: J. Clin. Invest. 118:2689-2693 (2008). doi:10.1172/JCI36536. our ability to combat them or even slow their relentless expansion. Cigarette smoke is the major cause of chronic obstructive pulmonary disease (COPD), which is characterized by chronic cough due to excessive mucus production (chronic bronchitis) and/or alveolar destruction leading to increased airspaces, known as centrilobular emphysema. COPD is the only major disease whose contribution to morbidity and mortality continues to increase, potentially displacing stroke as the third major worldwide cause of mortality by 2020 (1). This stark reality forces us to recognize the limitations of current disease paradigms and to search for answers that go beyond the traditional ways of thinking about COPD.

The classic paradigm of cigarette smokeinduced COPD has proposed that the alveolar destruction and enlargement is a direct consequence of inflammation and the associated imbalance in the extracellular matrix protease and antiprotease response, which leads to degradation of the elastin alveolar framework. This hypothesis continues to be revisited, as it has not led to significant therapeutic advances against the disease. Recent hypotheses have emphasized the resemblance of lung injury caused by cigarette smoke to the effects of aging in the lung, which results from the interaction between environmental stresses and homeostatic molecular and cellular processes involved in organismal protection (2). Cigarette smoke may thus co-opt some of the molecular signaling pathways involved in cellular sensing of environmental stresses, such as those triggered by starvation, radiation, or hypoxia, leading to progressive disruption 